\title{
Effect of self-care guidelines on knowledge and quality of life among faculty of nursing students with vaginal infection
}

\author{
Abstract \\ Vaginal infections are a global health problem for women at reproductive age. These \\ infections threat the women's health and have negative impacts on their QOL.
}

\begin{abstract}
Aim of the study: to evaluate the effect of self-care guidelines on knowledge, quality of life and practices, among faculty of nursing students with vaginal infection.
\end{abstract}

Subjects and methods: quasi experimental research design was used to conduct the study at Faculty of Nursing, El- Minia University. It included 214 female students selected by purposive sampling technique according to inclusion criteria, who suffer from vaginitis. The data were collected by using a self-administered questionnaire including sociodemographic data, knowledge, practice and WHOQOL scale.

Results: the prevalence of vaginal infection among female students constituted $25 \%$. The majority of studied students lacked the essential knowledge regarding vaginal infection in the pretest and has unsatisfactory practices score that consequently had negative impact on their quality of life. Meanwhile, after conduction of educational program it observed that there was a highly significant improvement in their knowledge, practice, as well as their quality of life among female students.

Conclusion: Self-care guidelines about vaginal infection were effective in improving the knowledge, practices as well as the quality of life for nursing students with vaginal infection.

Recommendation: Applying educational programs for adolescent females and their mothers to increase awareness about vaginal infection. Develop a special health center for adolescent females motivate them to seek prevention, early diagnosis and treatment of vaginal infection that may have positive impact on their future health and their QOL. Further research studies are needed to investigate and develop strategies to improve factors facing adolescent students to use reproductive health services.

Keywords: vaginal infection (vaginitis), quality of life (QOL), self-care guideline
Volume I0 Issue I - 2019

\author{
Safaa Ali Abdelnaem,' Shadia Hamido \\ Mohasib, ${ }^{2}$ Hoda Abd Elazim Mohamed ${ }^{3}$ \\ 'Assistant lecturer of Women Health and Obstetrics Nursing, \\ Faculty of Nursing, Minia University, Egypt \\ ${ }^{2}$ Professor of Maternity and Gynecological Health Nursing, \\ Faculty of Nursing, Ain shams university, Egypt \\ ${ }^{3}$ Associate Professor of Women Health and Obstetrics Nursing, \\ Faculty of Nursing, Minia University, Egypt
}

Correspondence: Safaa Ali Abdelnaem, Assistant lecturer of Women Health and Obstetrics Nursing, Faculty of Nursing Minia University, Minia, Egypt, Tel 01020626030,01285288843, Email Safaa_ali87@yahoo.com

Received: December 04, 2018 | Published: January 04, 2019

\section{Introduction}

The reproductive tract infection (RTI) or genital tract infection (GTI) is a global health problem which affects men, women, families and communities. Female adolescents alone have an estimated incidence of vaginitis of $10-25 \%$. Vaginitis is one of the most prevalent infections among RTIs, especially among adolescents. ${ }^{1}$ These infections threat the adolescent's health and may have severe consequences such as infertility, ectopic pregnancy, chronic pelvic pain, abortion and an increased risk of HIV transmission. Therefore, proper prevention and treatment of this infection are of great importance. ${ }^{2}$

Vaginitis is a general term that refers to inflammation of the vaginal wall. Almost $90 \%$ of the cases are caused by bacterial vaginosis, candidiasis, and trichomoniasis. Some predisposing factors for vaginitis include hormonal change (pregnancy, contraception, menopause), diabetes, long term using of antibiotics and corticosteroid, frequent douching, tight-fitting nylon pants, obesity; physical activity deficiency; high intake of sugar, carbohydrates, cola, and alcohol; low intake of dairy products; low vitamin $\mathrm{C}$; stress; sleep disorders; and low immune system. ${ }^{3}$
Nowadays, considering the importance of the self-care guidelines in health promotion, lifestyle modification, disease prevention is very important. Self-care consists of all activities related to disease management, health protection, prevention, and treatment which are conducted by individuals themselves. ${ }^{2}$ Knowledge and awareness about self-care guideline for vaginitis play crucial role in individuals' attitude, behavior and practice. Education of healthy practice skills and helping patients to acquire knowledge in order to make them follow self-care behaviors will result in disease prevention which improves their quality of life. ${ }^{4}$

Recurrent vaginal infection may impact negatively on adolescent female's social, personal and work relationships significantly affecting their quality of life. The primary role of the nurse in managing vaginal infections is to provide health education in order to modify the unhealthy behaviors and to prevent the occurrence as well as recurrence of vaginal infections to improve their quality of life.,

Aim of the study: The current research aimed to evaluate the effect of self-care guidelines on quality of life, knowledge and practices among faculty of nursing students with vaginal infection. 
Research hypothesis: Students who receive self-care guidelines regarding vaginal infection will have higher knowledge, good practices and better quality of life score than before they receive it.

\section{Subjects and methods}

Research Design: Quasi-experimental design (time series) has been utilized in this study.

Setting: The present study was conducted among nursing students in the Faculty of Nursing at El-Minia University.

Sample type: A purposive sample was used.

Sample size: the actual sample size (214) that was selected from the total number (854) after filling questionnaires (who are suffering from vaginitis).

Inclusion criteria: female students suffer from vaginal infection symptoms and female students willing to participate.

Exclusion criteria: female students have chronic disease as D.M, cancer, and female students have other infection except vaginal infection and use long term antibiotics.

\section{Tools of the study}

\section{Four tools for data collection were used in the present study}

The first tool (pretest): Arabic structured interviewing questionnaire was used that was developed by the researcher based on relevant literature, aim of the study and the data needed to be collected. It was divided into two parts.

Part (I): It was used to assess socio-demographic characteristic.

Part (II): It was used to assess vaginal infection characteristic.

The second tool: knowledge assessment tool (pre/post): It is a self-administered assessment tool developed by the researcher after revising relevant literature. It was designed to assess student's knowledge regarding vaginal infection that includes (11) questions MCQ. Total knowledge score was classified as: poor $(<50 \%)$, average (50\%-75\%), good (>75\%).

The third tool: practices assessment tool (pre/post): It is a selfadministered assessment tool developed by the researcher after revising relevant literature. It was designed to assess student's selfcare practices in dealing with vaginal infection, that includes (21) questions about the practices regarding vaginal infection. Total practices scores were classified as: unsatisfactory $(<60 \%)$ and satisfactory $(\geq 60 \%)$.

\section{The fourth tool: quality of life measuring scale (pre/post)}

World health organization quality of life (WHOQOL-BREF) (WHO, 1997): It contains 26 questions, including (2) general questions, and the remaining (24) questions divided into four domains: physical health (Q 3, 4, 10 and 15 to 18), psychological (Q 5, 6, 7, 11, 19 and 26), social relationships (Q 20 to 22), and environment (Q 8, 9, 12 to 14 and 23 to 25). WHOQOL-BREF scores are from 1 to 5 on a response scale. The WHOQOL-BREF scores are calculated according to an algorithm 26 that considers the number of answered questions in each of the domains and standardizes the scores of all domains from zero to 100 , with zero being the worst possible health condition and 100 being the best health condition. The algorithm inverts the score values for questions $(3,4$, and 26$)$ to calculate the final score.

\section{Ethical consideration}

All official permissions to carry out the study were secured from pertinent authorities. All students were informed about the importance and aim of this study. Oral consent was obtained from all the participants. All students were informed that their participation is voluntary and their rights to withdraw at any time, and confidentiality of the information obtained. Also, the students were informed that the collected data would be used only for the purpose of the present study, as well as for their benefit.

\section{Procedure}

An official written approval letter clarifying the purpose of the present study was approved from the Dean of the Faculty of Nursing at Minia University, Vice dean of student's affairs, The head of each department, Ethical committee and Post graduate committee in faculty of nursing as an approval for data collection to conduct this study.

The current study was achieved through three phases: assessment phase (pre-test), implementation phase (conducting education program), follow up and evaluation phase (post-test).

\section{Assessment phase (pre-test)}

a. During assessment phase the researcher held the first meeting with each academic level in their faculty during one of their free classes or between lectures to introduce herself and briefly explained the nature and the purpose of the study. They were informed that participation in this study was voluntary and they had the right to withdraw at any time. Oral approval of students to share in this study was achieved.

b. After obtaining the acceptance from the students to participate in the current study, the researcher provided an overview and clarification about the assessment tools questions to the whole class. Then, the self-administered questionnaire was distributed to each student to assess the data related to socio-demographic data and the presence of vaginal infection symptoms. The questionnaire took about 15 minutes to be completed.

c. The second meeting was conducted after analysis of the first self-administered questionnaire that was distributed in the first meeting to select only the students who suffer from vaginal infection symptoms. The researcher distributed the pretest questionnaire on the selected students who suffer from vaginal infection to assess their knowledge and their practices as well as their quality of life regarding vaginal infection. It was filled by the students in a time ranged from 20 to 25 minutes to be completed.

\section{Implementation phase (conducting education program)}

i. After assessing the students' knowledge, practices and their quality of life regarding vaginal infection by pretest selfadministered questionnaire. The total sample (214) was divided into small groups 8 groups each group ranged from 25-30 students. The total number of educational program sessions was 16 sessions, two sessions for each group (2 days/week), each session was conducted for one hour in the time they are free by organization with the students and with the staff members for each department.

ii. Health education sessions were given to the students in the form 
of lectures and group discussion by using audio-visual aids, it emphasized on improving student's knowledge and self-care practices. An additional 15 minutes were assigned at the end of the lecture for an open discussion with the students about this topic and feedback from the students was obtained to ensure that the students got the maximum benefits.

iii. Booklet and brochures containing information about vaginal infection were distributed to students at the end of session for students who were selected based on inclusion criteria. Also, the researcher communicated with students via telephone call for instruction and reinforcement.

\section{Follow up and evaluation phase (post-test)}

a. Three evaluations were done for each student. The first one was at the beginning of the study as a baseline data (pre-test). The second evaluation was conducted after one month from the education program. The third evaluation was conducted after three months from the second evaluation in order to detect the effect of self-care guidelines on quality of life, knowledge and practices of students regarding vaginal infection (follow up post-test).

b. The effect of self-care guideline was done through comparing between the pretest and posttest that were conducted after one month and three months of intervention to assess their knowledge and their practices regarding vaginal infection as well as their quality of life were assessed. The data was collected through a period of 8 months from October 2017 to May 2018.

\section{Statistical analysis}

The collected data were organized; categorized, analyzed using the statistical package for social studies (SPSS). Data were presented using descriptive statistics in the form of frequencies and percentages for qualitative variables, mean and standard deviations for quantitative variables. The statistical test such as chi-square test was to determine relation between qualitative data and r-test was used. Statistical significance difference was considered when $p$-value $\leq 0.05$, and high significance when $p$-value $\leq 0.001$ and no statistical significance difference was considered when $p$-value $>0.05$.

\section{Results}

Table 1 illustrates distribution of the studied students regarding to their socio-demographic characteristics. Regarding Students' age it ranges from 18-22 years old with mean age 19.9 \pm 1.1 years. Regarding academic level, more than one third of the students $(34.2 \%)$ at $2^{\text {nd }}$ academic level. Concerning to residence, more than three quarters of the students $(82.7 \%)$ were living in rural areas. Regarding marital status, the majority of the students $(94.4 \%)$ were single. As regard mother's education, it is obvious that more than one third $(39.3 \%)$ were illiterate. In relation to socioeconomic status the majority of students $(87.4 \%)$ had moderate income.

Regarding distribution of the prevalence of vaginal infection symptoms among the total number of students at all academic levels, It was observed that about one quarter (25\%) said yes they suffer from vaginal infection, while $(19 \%)$ said they don't suffer from vaginal infection symptoms, and more than half of study sample (56 \%) don't know if they had vaginal infection symptoms or not (Figure 1).
Table I Distribution of students' regarding to their socio-demographic characteristics $(n=2 \mid 4)$

\begin{tabular}{|c|c|c|}
\hline Socio-demographic characteristics & $\mathrm{No}=214$ & $\%$ \\
\hline \multicolumn{3}{|l|}{ Age/year } \\
\hline $18-<20$ & 83 & 38.8 \\
\hline $20-<22$ & 114 & 53.3 \\
\hline$\geq 22$ & 17 & 7.9 \\
\hline Mean \pm SD & \multicolumn{2}{|c|}{$19.9 \pm 1.1$ years } \\
\hline \multicolumn{3}{|l|}{ Academic Level } \\
\hline$I^{\text {st }}$ & 61 & 28.5 \\
\hline $2^{\text {nd }}$ & 73 & 34.2 \\
\hline $3^{\text {rd }}$ & 57 & 26.6 \\
\hline $4^{\text {th }}$ & 23 & 10.7 \\
\hline \multicolumn{3}{|l|}{ Residence } \\
\hline Urban & 37 & 17.3 \\
\hline Rural & 177 & 82.7 \\
\hline \multicolumn{3}{|l|}{ Marital status } \\
\hline Single & 202 & 94.4 \\
\hline Married & 12 & 5.6 \\
\hline \multicolumn{3}{|l|}{ Mother's education } \\
\hline Illiterate & 84 & 39.3 \\
\hline Read/Write & 45 & 21 \\
\hline Secondary & 51 & 23.8 \\
\hline University & 34 & 15.9 \\
\hline \multicolumn{3}{|l|}{ Socioeconomic Status } \\
\hline Low & 22 & 10.3 \\
\hline Moderate & 187 & 87.4 \\
\hline High & 5 & 2.3 \\
\hline
\end{tabular}

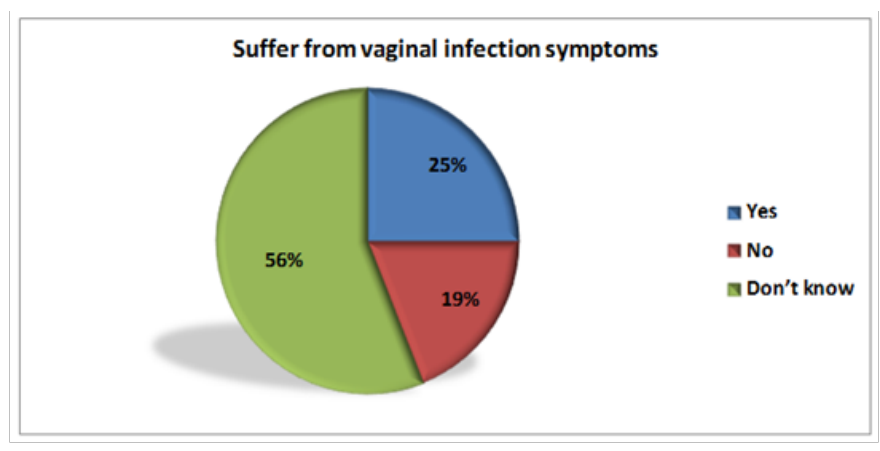

Figure I Distribution of students related to the prevalence of vaginal infection.

Table 2 shows distribution of the students regarding to vaginal infection characteristics. It was observed that, more than one third of students $(41.6 \%)$ suffer from candidiasis and only $(3.7 \%)$ suffer from bacterial vaginosis. Around two thirds of students $(61.7 \%)$ the period 
of their symptoms last less than one week. Regarding to the number of vaginal infection episodes per year, around two thirds of students $(63.1 \%)$ the symptoms occur three times or more per year. In relation to the kind of treatment used during suffering of infection, and more than half of students (57.0\%) not treated. On the other hand, more than half of students $(52.8 \%)$ can't tell any person when expose to vaginal infection, and $(22.4 \%)$ prefer to tell their mother about complain.

Table 2 Distribution of students regarding to their vaginal infection characteristics $(n=2 \mid 4)$

Vaginal infection characteristics $\quad \mathrm{N}=2 \mid 4$

\section{Symptoms from the following you suffer}

Itching, burning, swelling, malodorous, abnormal discharge and leucorrhea (General symptoms)

Thick white vaginal discharge as small pieces like white cheese (Candida symptoms)

Thin, white (milky) or gray vaginal discharge, and fishy odor (Bacterial vaginosis)

Itching, burning, swelling, malodorous, abnormal discharge and leucorrhea + Thick white vaginal discharge like white cheese.

Thick white vaginal discharge as small pieces like white cheese + Thin, white (milky) or gray vaginal discharge, and fishy odor.

Period of vaginal infection symptoms from the beginning at this episode

Less than one week

One week

More than one week

Number of vaginal infection episodes per year

Once

Two times

Three times or more

\section{Kind of treatment used}

Medication

Douching

Cotton underwear

Not treated

\section{Students action taking}

Go to private clinic to seek treatment

Tell my mother about my complain

Ask my sister about my complain

\section{Vaginal infection characteristics}

Ask my friends about my complain

Cannot tell any person

Others (pharmacist, asked doctor in my work)

Barriers to seek gynecologist when exposed to vaginal infection

Shyness

Tradition

No special girl's center

The belief that this is a natural 
As regard the barriers that prevent female students from seeking gynecologist when exposed to vaginal infection, it was observed that the shyness was the most pronounced barrier (58.0\%). The belief that this is natural come the next barrier $(28.5 \%)$, followed by there is no special girl's center $(10.3 \%)$, and the tradition $(4.2 \%)$.

Regarding distribution of the students regarding to their sources of knowledge about vaginal infection. It noticed that more than half $(59.8 \%)$ of them selected the mother as a source of information about infection, followed by friends and relatives (49.1\%), (39.3\%) from mass media (T.V, Internet), physician $(27.1 \%)$, nurse $(25.7 \%)$, $(23.8 \%)$ school, university, books, and the last $(9.8 \%)$ from others sources (pharmacist) (Figure 2).

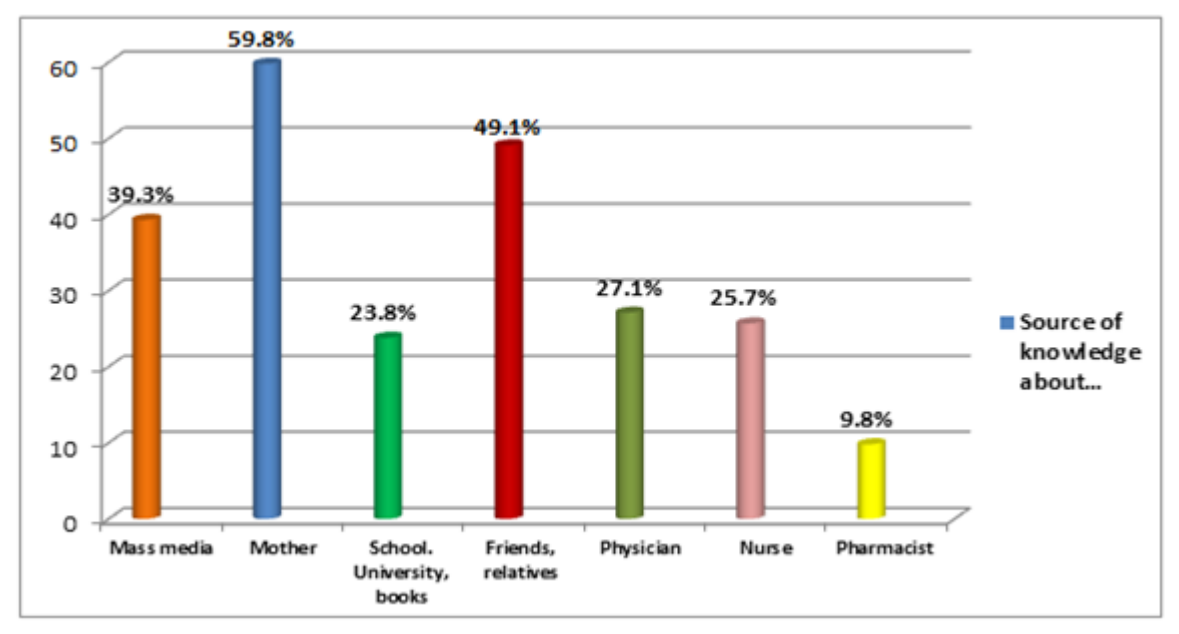

Figure 2 The students' sources of knowledge about vaginal infection.

Table 3 illustrates distribution of students regarding to their total knowledge about vaginal infection pre and post educational program. It showed that, there was a significant improvement in total knowledge

about vaginal infection among students in post-test as compared to pretest assessment $(\mathrm{p}<0.0001)$.

Table 3 Distribution of students regarding to their total knowledge about vaginal infection pre and post educational program $(n=2 / 4)$

\begin{tabular}{lllllllll}
\hline \multirow{2}{*}{ Total knowledge } & \multicolumn{9}{l}{ Pretest } & \multicolumn{3}{l}{ Post I Month } & \multicolumn{2}{l}{ Post 3 } & Months & \multirow{2}{*}{$\mathbf{X}^{2}$} & P-Value \\
\cline { 2 - 7 } & No & $\%$ & No & $\%$ & No & $\%$ & & \\
\hline Poor $(<50 \%)$ & 129 & 60.3 & 13 & 6.1 & 21 & 9.8 & & \\
Moderate (50-75\%) & 75 & 35 & 71 & 33.2 & 97 & 45.3 & 256.597 & $.000 * *$ \\
Good (75\%) & 10 & 4.7 & 130 & 60.7 & 96 & 44.9 & & \\
Mean \pm SD & $14.2 \pm 4.6$ & $23.0 \pm 4.6$ & $21.4 \pm 4.9$ & F 215.345 & $.000 * *$ \\
\hline
\end{tabular}

**Highly statistically significant difference

Table 4 illustrates distribution of students' related to their total practice regarding vaginal infection. It was noticed that, there was a significant improvement in total practice regarding vaginal infection among the students in post-test as compared to pretest assessment $(p<0.0001)$.

Table 4 Distribution of nursing students' pre and post one month and after 3 months regarding to their totally practice $(n=214)$

\begin{tabular}{|c|c|c|c|c|c|c|c|c|}
\hline \multirow{2}{*}{ Total practice } & \multicolumn{2}{|c|}{ Pretest } & \multicolumn{2}{|c|}{ Post I Month } & \multicolumn{2}{|c|}{ Post 3 Months } & \multirow{2}{*}{$\mathbf{X}^{2}$} & \multirow{2}{*}{ P-Value } \\
\hline & No & $\%$ & No & $\%$ & No & $\%$ & & \\
\hline Unsatisfactory & 97 & 45.3 & 42 & 19.6 & 45 & 21 & \multirow{2}{*}{43.713} & \multirow{2}{*}{$.000 * *$} \\
\hline Satisfactory & 117 & 54.7 & 172 & 80.4 & 169 & 79 & & \\
\hline Mean $\pm S D$ & \multicolumn{2}{|c|}{$25.4 \pm 5.8$} & \multicolumn{2}{|c|}{$29.6 \pm 4.8$} & \multicolumn{2}{|c|}{$29.8 \pm 5.3$} & F 44.052 & $.000 * *$ \\
\hline
\end{tabular}

\footnotetext{
** Highly statistically significant difference
}

Table 5 reveals that there is a highly significant difference between the mean score of total quality of life of the students in pretest and posttest after 1 month and 3 month. It showed a significant improvement in of quality of life among students in post-test as compared to pretest assessment $(p<0.001)$ (Figure 3). 
Table 5 Mean and standard deviation of total quality of life scores among students' pre, post one month and after 3 months $(\mathrm{N}=2 \mid 4)$

\begin{tabular}{|c|c|c|c|c|c|}
\hline \multirow{2}{*}{ Quality of life domains } & Pretest & Post I Month & Post 3 Months & \multirow{2}{*}{$\mathbf{F}$} & \multirow{2}{*}{$P$ - value } \\
\hline & Mean士SD & Mean $\pm S D$ & Mean $\pm S D$ & & \\
\hline Overall QOL \& general health & $6.8 \pm 1.8$ & $7.4 \pm 1.3$ & $7.5 \pm 1.4$ & 12.663 & $.000 * *$ \\
\hline Physical & $22.9 \pm 4.4$ & $24.3 \pm 3.7$ & $24.4 \pm 3.6$ & 9.35 & $.000 * *$ \\
\hline Psychological & $18.3 \pm 3.9$ & $19.5 \pm 3.2$ & $19.6 \pm 3.6$ & 9.223 & $.000 * *$ \\
\hline Social & $7.4 \pm 1.6$ & $8.0 \pm 1.3$ & $7.9 \pm 1.4$ & 6.327 & $.002 * *$ \\
\hline Environmental & $24.5 \pm 5.1$ & $26.5 \pm 4.5$ & $27.3 \pm 4.5$ & 19.168 & $.000 * *$ \\
\hline Total Qol & $80.0 \pm 13.4$ & $85.4 \pm 11.3$ & $86.8 \pm 11.7$ & 18.484 & $.000 * *$ \\
\hline
\end{tabular}

**Highly statistically significant difference

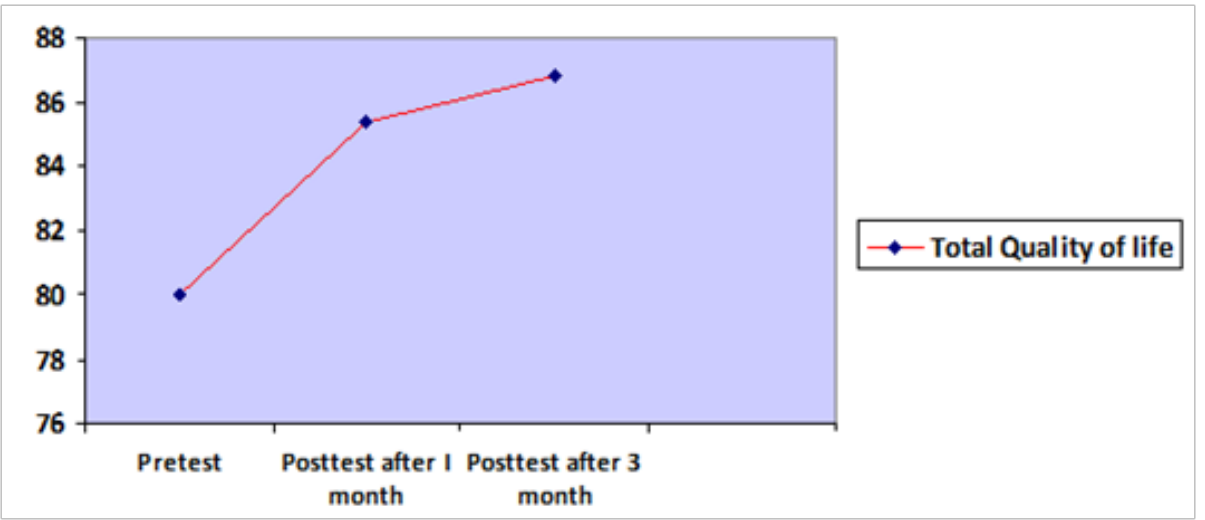

Figure 3 Distribution of students regarding their total quality of life scores in pretest and post I month and post 3 months.

Table 6 illustrates the relationship between students' total knowledge with their socio-demographic characteristics. It was noticed that there was a highly statistically significant relation between the students' total score of pretest knowledge as regards vaginitis with their age, academic level, residence and mother's education ( $\mathrm{P}$ value $<0.001,0.001,0.02$, and, 0.04), respectively. While in post- test after 1 month, there was no statistically significant $(\mathrm{p}>0.05)$ relation between students' total knowledge score with students' sociodemographic data. After 3 months there was a highly statistically significant relation between the students' total knowledge score with their age and academic level $(\mathrm{P}<0.005,0.01)$, respectively.

Table 6 Relation between total knowledge of student's pre and post one month and 3 months with their socio-demographic characteristics ( $\mathrm{n}=2 \mathrm{I} 4$ )

\begin{tabular}{|c|c|c|c|c|c|c|c|c|c|}
\hline \multirow{4}{*}{$\begin{array}{l}\text { Socio-demographic } \\
\text { characteristics }\end{array}$} & \multicolumn{9}{|c|}{ Total knowledge } \\
\hline & \multicolumn{3}{|l|}{ Pretest } & \multicolumn{3}{|c|}{ Post I Month } & \multicolumn{3}{|c|}{ Post 3 Months } \\
\hline & $\begin{array}{l}\text { Poor } \\
(N=\mid 29)\end{array}$ & $\begin{array}{l}\text { Average } \\
(\mathrm{N}=75)\end{array}$ & $\begin{array}{l}\text { Good } \\
(N=\mid 0)\end{array}$ & $\begin{array}{l}\text { Poor } \\
(N=\mid 3)\end{array}$ & $\begin{array}{l}\text { Average } \\
(\mathbf{N}=7 \text { I) }\end{array}$ & $\begin{array}{l}\text { Good } \\
(N=\mid 30)\end{array}$ & $\begin{array}{l}\text { Poor } \\
(N=2 I)\end{array}$ & $\begin{array}{l}\text { Average } \\
(\mathrm{N}=97)\end{array}$ & $\begin{array}{l}\text { Good } \\
(N=96)\end{array}$ \\
\hline & $\%$ & $\%$ & $\%$ & $\%$ & $\%$ & $\%$ & $\%$ & $\%$ & $\%$ \\
\hline \multicolumn{10}{|l|}{ Age/year } \\
\hline $18-<20$ & 49.6 & 25.3 & 0 & 38.5 & 46.5 & 34.6 & 61.9 & 37.1 & 35.4 \\
\hline $19-<22$ & 45 & 64 & 80 & 46.1 & 43.6 & 59.2 & 33.3 & 56.6 & 57.3 \\
\hline$\geq 22$ & 5.4 & 10.7 & 20 & 15.4 & 9.9 & 6.2 & 4.8 & 9.3 & 7.3 \\
\hline$X^{2}(P-$ value $)$ & \multicolumn{3}{|c|}{$19.469\left(.001^{* *}\right)$} & \multicolumn{3}{|c|}{$5.646(.227)$} & \multicolumn{2}{|c|}{$5.642(.228)$} & \\
\hline \multicolumn{10}{|l|}{ Academic level } \\
\hline$\left.\right|^{\text {st }}$ & 39.5 & 13.3 & 0 & 30.8 & 39.4 & 23.1 & 61.9 & 30.9 & 19.8 \\
\hline $2^{\text {nd }}$ & 31.8 & 37.3 & 40 & 38.5 & 28.2 & 37.7 & 23.8 & 30.9 & 40.6 \\
\hline $3^{\text {rd }}$ & 22.5 & 32 & 40 & 30.8 & 19.7 & 28.5 & 9.5 & 27.8 & 27.1 \\
\hline $4^{\text {th }}$ & 6.2 & 17.3 & 20 & 0 & 12.7 & 10.8 & 4.8 & 10.3 & 12.5 \\
\hline $\mathrm{X}^{2}(\mathrm{P}-$ value $)$ & \multicolumn{3}{|c|}{$23.568\left(.00 I^{* *}\right)$} & \multicolumn{3}{|c|}{$8.669(.193)$} & \multicolumn{2}{|c|}{ I $6.248(.01 *)$} & \\
\hline
\end{tabular}


Table Continued...

\begin{tabular}{|c|c|c|c|c|c|c|c|c|c|}
\hline \multirow{4}{*}{$\begin{array}{l}\text { Socio-demographic } \\
\text { characteristics }\end{array}$} & \multicolumn{9}{|c|}{ Total knowledge } \\
\hline & \multicolumn{3}{|l|}{ Pretest } & \multicolumn{3}{|c|}{ Post I Month } & \multicolumn{3}{|c|}{ Post 3 Months } \\
\hline & $\begin{array}{l}\text { Poor } \\
(N=\mid 29)\end{array}$ & $\begin{array}{l}\text { Average } \\
(\mathrm{N}=75)\end{array}$ & $\begin{array}{l}\text { Good } \\
(N=\mid 0)\end{array}$ & $\begin{array}{l}\text { Poor } \\
(N=\mid 3)\end{array}$ & $\begin{array}{l}\text { Average } \\
(\mathbf{N}=7 \text { I) }\end{array}$ & $\begin{array}{l}\text { Good } \\
(N=\mid 30)\end{array}$ & $\begin{array}{l}\text { Poor } \\
(N=2 I)\end{array}$ & $\begin{array}{l}\text { Average } \\
(\mathrm{N}=97)\end{array}$ & $\begin{array}{l}\text { Good } \\
(N=96)\end{array}$ \\
\hline & $\%$ & $\%$ & $\%$ & $\%$ & $\%$ & $\%$ & $\%$ & $\%$ & $\%$ \\
\hline \multicolumn{10}{|l|}{ Residence } \\
\hline Urban & 20.2 & 9.3 & 40 & 23.1 & 18.3 & 16.2 & 14.3 & 22.7 & 12.5 \\
\hline Rural & 79.8 & 90.7 & 60 & 76.9 & 81.7 & 83.8 & 85.7 & 77.3 & 87.5 \\
\hline$X^{2}(P-$ value $)$ & \multicolumn{3}{|l|}{$7.667(.02 *)$} & \multicolumn{2}{|c|}{$.473(.789)$} & & \multicolumn{2}{|c|}{$3.644(.162)$} & \\
\hline \multicolumn{10}{|l|}{ Marital status } \\
\hline Single & 94.6 & 93.3 & 100 & 100 & 91.5 & 95.4 & 95.2 & 93.8 & 94.8 \\
\hline Married & 5.4 & 6.7 & 0 & 0 & 8.5 & 4.6 & 4.8 & 6.2 & 5.2 \\
\hline$X^{2}(P-$ value $)$ & \multicolumn{3}{|l|}{$.761(.684)$} & \multicolumn{3}{|c|}{$2.098(.350)$} & \multicolumn{2}{|c|}{$.119(.942)$} & \\
\hline \multicolumn{10}{|l|}{ Mother's education } \\
\hline Illiterate & 38 & 44 & 20 & 30.8 & 39.4 & 40 & 42.9 & 28.9 & 49 \\
\hline Read/Write & 24.8 & 14.7 & 20 & 30.8 & 25.4 & 17.7 & 23.8 & 22.7 & 18.8 \\
\hline Secondary & 24.8 & 24 & 10 & 23.1 & 19.7 & 26.2 & 23.8 & 30.9 & 16.7 \\
\hline University & 12.4 & 17.3 & 50 & 15.4 & 15.5 & 16.2 & 9.5 & 17.5 & 15.6 \\
\hline$X^{2}(P-$ value $)$ & \multicolumn{3}{|l|}{$13.016(.04 *)$} & \multicolumn{3}{|c|}{$2.979(.81 \mathrm{I})$} & \multicolumn{2}{|c|}{$10.300(.113)$} & \\
\hline \multicolumn{10}{|l|}{ Socioeconomic status } \\
\hline Low & 10.1 & 12 & 0 & 15.4 & 9.9 & 10 & 4.8 & 6.2 & 15.7 \\
\hline Moderate & 89.1 & 82.7 & 100 & 84.6 & 90.1 & 86.2 & 95.2 & 89.7 & 83.3 \\
\hline High & 0.8 & 5.3 & 0 & 0 & 0 & 3.8 & 0 & 4.1 & I \\
\hline $\mathrm{X}^{2}(\mathrm{P}-$ value $)$ & \multicolumn{3}{|l|}{$6.130(.190)$} & \multicolumn{3}{|c|}{$3.678(.45 \mathrm{I})$} & \multicolumn{2}{|c|}{$7.765(.101)$} & \\
\hline
\end{tabular}

NS, No significant difference

* Statistically significant difference

** Highly statistically significant difference

Table 7 illustrates the relationship between the students' total level (P value $<0.03$ and 0.002 ), respectively. While in post-test after practices with their socio-demographic characteristics. It was noticed 1month and 3 months it was noticed that there was no statistically that there was a highly statistically significant relation between the significant ( $\mathrm{p}>0.05$ ) relation between students' total practices scores students' total practices score of pretest with their age and academic with their socio-demographic characteristics.

Table 7 Relation between total practice of students pre and post one month and after 3 months with their socio-demographic characteristics ( $=2$ I 4 )

\begin{tabular}{|c|c|c|c|c|c|c|}
\hline \multirow{4}{*}{$\begin{array}{l}\text { Socio- } \\
\text { demographic } \\
\text { characteristics }\end{array}$} & \multicolumn{6}{|c|}{ Total Practice Scores } \\
\hline & \multicolumn{2}{|l|}{ Pretest } & \multicolumn{2}{|l|}{ Post I Month } & \multicolumn{2}{|c|}{ Post 3 Months } \\
\hline & $\begin{array}{l}\text { Satisfactory } \\
(n=\mid I 7)\end{array}$ & $\begin{array}{l}\text { Unsatisfactory } \\
(n=97)\end{array}$ & $\begin{array}{l}\text { Satisfactory } \\
(n=\mid 72)\end{array}$ & $\begin{array}{l}\text { Unsatisfactory } \\
(n=42)\end{array}$ & $\begin{array}{l}\text { Satisfactory } \\
(n=169)\end{array}$ & $\begin{array}{l}\text { Unsatisfactory } \\
(n=45)\end{array}$ \\
\hline & $\%$ & $\%$ & $\%$ & $\%$ & $\%$ & $\%$ \\
\hline \multicolumn{7}{|l|}{ Age/year } \\
\hline $18-<20$ & 29.1 & 50.5 & 40.1 & 33.3 & 37.9 & 42.2 \\
\hline $20-<22$ & 62.4 & 42.3 & 52.9 & 54.8 & 52.6 & 55.6 \\
\hline$\geq 22$ & 8.5 & 7.2 & 7 & 11.9 & 9.5 & 2.2 \\
\hline$X^{2}(P-$ value $)$ & $10.445\left(.005^{* *}\right)$ & & $\mathrm{I} .454(.483)$ & & $2.578(.276)$ & \\
\hline
\end{tabular}


Table Continued...

\begin{tabular}{|c|c|c|c|c|c|c|}
\hline \multirow{4}{*}{$\begin{array}{l}\text { Socio- } \\
\text { demographic } \\
\text { characteristics }\end{array}$} & \multicolumn{6}{|c|}{ Total Practice Scores } \\
\hline & \multicolumn{2}{|l|}{ Pretest } & \multicolumn{2}{|l|}{ Post I Month } & \multicolumn{2}{|c|}{ Post 3 Months } \\
\hline & $\begin{array}{l}\text { Satisfactory } \\
(n=\mid 17)\end{array}$ & $\begin{array}{l}\text { Unsatisfactory } \\
(n=97)\end{array}$ & $\begin{array}{l}\text { Satisfactory } \\
(n=\mid 72)\end{array}$ & $\begin{array}{l}\text { Unsatisfactory } \\
(n=42)\end{array}$ & $\begin{array}{l}\text { Satisfactory } \\
(n=169)\end{array}$ & $\begin{array}{l}\text { Unsatisfactory } \\
(n=45)\end{array}$ \\
\hline & $\%$ & $\%$ & $\%$ & $\%$ & $\%$ & $\%$ \\
\hline \multicolumn{7}{|l|}{ Academic Level } \\
\hline$\left.\right|^{\text {st }}$ & 17.9 & 41.2 & 30.2 & 21.4 & 26 & 37.8 \\
\hline $2^{\text {nd }}$ & 38.5 & 28.9 & 36 & 26.2 & 36.7 & $24 . .4$ \\
\hline $3^{\text {rd }}$ & 32.5 & 19.6 & 22.7 & 42.9 & 24.9 & 33.3 \\
\hline $4^{\text {th }}$ & II.I & 10.3 & II & 9.5 & 12.4 & 4.4 \\
\hline $\mathrm{X}^{2}(\mathrm{P}-$ value $)$ & I4.862 (.002**) & & 7.115 (.068) & & $6.346(.096)$ & \\
\hline \multicolumn{7}{|l|}{ Residence } \\
\hline Urban & 17.1 & 17.5 & 16.3 & 21.4 & 17.8 & 15.6 \\
\hline Rural & 82.9 & 82.5 & 83.7 & 78.6 & 82.2 & 84.4 \\
\hline $\mathrm{X}^{2}(\mathrm{P}-$ value $)$ & $.007(.934)$ & & $.626(.429)$ & & $.120(.729)$ & \\
\hline \multicolumn{7}{|l|}{ Marital status } \\
\hline Single & 94.9 & 93.8 & 94.2 & 95.2 & 94.1 & 95.6 \\
\hline Married & 5.1 & 6.2 & 5.8 & 4.8 & 5.9 & 4.4 \\
\hline$X^{2}(P-$ value $)$ & $.112(.738)$ & & .071 (.790) & & $.146(.703)$ & \\
\hline \multicolumn{7}{|c|}{ Mother's education } \\
\hline Illiterate & 38.5 & 40.2 & 40.1 & 35.7 & 37.9 & 44.4 \\
\hline Read/Write & 23.1 & 18.6 & 19.8 & 26.2 & 22.5 & 15.6 \\
\hline Secondary & 23.1 & 24.7 & 23.3 & 26.2 & 23.7 & 24.4 \\
\hline University & 15.4 & 16.5 & 16.9 & 11.9 & 16 & 15.6 \\
\hline $\mathrm{X}^{2}(\mathrm{P}-\mathrm{value})$ & $.659(.883)$ & & $1.473(.689)$ & & $1.216(.749)$ & \\
\hline \multicolumn{7}{|c|}{ Socioeconomic Status } \\
\hline Low & 12 & 8.2 & 10.5 & 9.5 & 9.5 & 13.3 \\
\hline Moderate & 86.3 & 88.7 & 87.2 & 88.1 & 88.2 & 84.4 \\
\hline High & 1.7 & 3.1 & 2.3 & 2.4 & 2.4 & 2.2 \\
\hline$X^{2}(P-$ value $)$ & I.I8I (.554) & & $.033(.984)$ & & $.576(.750)$ & \\
\hline
\end{tabular}

NS, No Significant difference

* Statistically significant difference

** Highly statistically significant difference

Table 8 illustrates the relation between the students quality of life with their socio-demographic characteristics. It was noticed that, there was a highly statistically significant relation between the students' total QOL score of pretest with their socioeconomic status $(\mathrm{P}<0.01)$. In post-test after 1 month, it was noticed that there was statistically significant relation between the students' total QOL score with their academic level and socioeconomic status $(\mathrm{P}$ value $<0.001$ and 0.001 ), respectively. In post-test after 3 months, it noticed that there was statistically significant relation between the students' total QOL scores with their socioeconomic status $(\mathrm{P}<0.01)$.

Table 9 illustrates the relation between total knowledge of the students and their total practices. It was observed that there was highly statistical significant relation between total knowledge of the students with their practices, $(\mathrm{P}$ value $<0.01)$.

Table 10 illustrates the relation between total knowledge of the students as regards to their total quality of life. It was observed that there was no statistically significant relation between total knowledge of the students with their total quality of life.

Table 11 illustrates the relation between the total quality of life of students with their total knowledge and their total practice. It was observed that there was positive correlation between their quality of life and their practices, pre and post educational program ( $p$ value 0.000 ). While there were no significant correlation between their quality of life and their knowledge, pre and post educational program ( $\mathrm{p}$ value $>0.01$ ). 
Table 8 Relation between total quality of life scores among students pre and post one month and after 3 months with their socio-demographic characteristics $(n=214)$

\begin{tabular}{|c|c|c|c|}
\hline \multirow{3}{*}{ Socio-demographic characteristics } & \multicolumn{3}{|l|}{ Total QOL } \\
\hline & Pretest & Post I Month & Post 3 Months \\
\hline & Mean \pm SD & Mean \pm SD & Mean士SD \\
\hline \multicolumn{4}{|l|}{ Age/year } \\
\hline $18-<20$ & $87.7 \pm 11.1$ & $87.7 \pm 11.1$ & $87.1 \pm 11.1$ \\
\hline $20-<22$ & $84.2 \pm 10.9$ & $84.2 \pm 10.9$ & $86.7 \pm 11.7$ \\
\hline$\geq 22$ & $83.1 \pm 13.2$ & $83.1 \pm 13.2$ & $86.2 \pm \mid 5.1$ \\
\hline$F(P-$ value $)$ & $2.700(.07)$ & $2.700(.07)$ & $.05(.949)$ \\
\hline \multicolumn{4}{|l|}{ Academic Level } \\
\hline$\left.\right|^{s t}$ & $80.6 \pm 12.2$ & $88.6 \pm 10.2$ & $86.8 \pm \mid I .4$ \\
\hline $2^{\text {nd }}$ & $81.1 \pm 13.5$ & $86.5 \pm 11.0$ & $88.2 \pm 10.9$ \\
\hline $3^{\text {rd }}$ & $77.8 \pm 14.6$ & $80.6 \pm 12.5$ & $83.9 \pm 13.7$ \\
\hline $4^{\text {th }}$ & $80.4 \pm 12.9$ & $85.7 \pm 8.2$ & $89.4 \pm 8.6$ \\
\hline $\mathrm{F}(\mathrm{P}-$ value $)$ & $.726(.537)$ & $5.657(.001 * *)$ & $1.856(.138)$ \\
\hline \multicolumn{4}{|l|}{ Residence } \\
\hline Urban & $80.1 \pm 12.9$ & $85.2 \pm 12.5$ & $86.1 \pm 13.2$ \\
\hline Rural & $80.0 \pm 13.5$ & $85.5 \pm 11.1$ & $86.9 \pm 11.4$ \\
\hline $\mathrm{F}(\mathrm{P}-$ value $)$ & $.001(.970)$ & $.148(.883)$ & $.161(.688)$ \\
\hline \multicolumn{4}{|l|}{ Marital status } \\
\hline Single & $79.9 \pm 13.3$ & $85.3 \pm 11.5$ & $86.7 \pm 11.9$ \\
\hline Married & $81.6 \pm 14.6$ & $87.2 \pm 8.2$ & $87.8 \pm 8.7$ \\
\hline $\mathrm{F}(\mathrm{P}-$ value $)$ & $.170(680)$ & $.544(.587)$ & $.293(.770)$ \\
\hline \multicolumn{4}{|l|}{ Mother's education } \\
\hline Illiterate & $77.7 \pm 13.4$ & $83.7 \pm 12.0$ & $85.5 \pm 11.7$ \\
\hline Read/Write & $80.9 \pm 12.2$ & $87.4 \pm 11.1$ & $88.7 \pm 10.1$ \\
\hline Secondary & $81.7 \pm 15.2$ & $86.6 \pm 11.8$ & $86.5 \pm 12.9$ \\
\hline University & $82.0 \pm 11.5$ & $85.3 \pm 8.6$ & $87.8 \pm 11.7$ \\
\hline $\mathrm{F}(\mathrm{P}$-value $)$ & $1.420(.238)$ & $1.317(.270)$ & $.813(.488)$ \\
\hline \multicolumn{4}{|l|}{ Socioeconomic status } \\
\hline Low & $73.9 \pm 13.1$ & $76.8 \pm 14.5$ & $80.6 \pm 15.7$ \\
\hline Moderate & $80.4 \pm \mid 3.1$ & $86.4 \pm 10.6$ & $87.5 \pm I I .1$ \\
\hline High & $92.4 \pm 13.7$ & $89.0 \pm 6.9$ & $6.9 \pm 3.1$ \\
\hline $\mathrm{F}(\mathrm{P}$-value $)$ & $4.643(.01 *)$ & $7.802\left(.00 I^{* *}\right)$ & $3.47 \mathrm{I}(.03 *)$ \\
\hline
\end{tabular}

NS, No Significant difference

* Statistically significant difference

** Highly statistically significant difference

Table 9 Relation between total knowledge of the students with their total practices; pre and post educational program $(n=2 \mid 4)$

\begin{tabular}{|c|c|c|c|c|c|c|c|c|c|}
\hline \multirow{3}{*}{$\begin{array}{l}\text { Total } \\
\text { practice } \\
\text { scores }\end{array}$} & \multicolumn{9}{|c|}{ Total knowledge Scores } \\
\hline & \multicolumn{3}{|l|}{ Pretest } & \multicolumn{3}{|c|}{ Post I Month } & \multicolumn{3}{|c|}{ Post 3 Months } \\
\hline & Poor & Average & Good & Poor & Average & Good & Poor & Average & Good \\
\hline Satisfactory & $62(48.1 \%)$ & $47(62.7 \%)$ & $8(80.00 \%)$ & $5(38.50 \%)$ & $58(81.70 \%)$ & 109 (83.80\%) & $12(57.10 \%)$ & 77 (79.40\%) & $80(83.30 \%)$ \\
\hline Unsatisfactory & 67 (51.90\%) & $28(37.30 \%)$ & $2(20.00 \%)$ & $8(31.50 \%)$ & $13(18.30 \%)$ & $21(16.20 \%)$ & $9(42.90 \%)$ & $20(20.60 \%)$ & $16(16.70 \%)$ \\
\hline $\mathrm{X}^{2}(\mathrm{P}-$ value $)$ & \multicolumn{3}{|l|}{$6.797(.03)^{*}$} & \multicolumn{3}{|c|}{$15.549(.000)^{* *}$} & \multicolumn{3}{|l|}{$7.135(.03)^{*}$} \\
\hline
\end{tabular}

* Statistically significant difference

** Highly statistically significant difference

Citation: Abdelnaem SA, Mohasib SH, Mohamed HAE. Effect of self-care guidelines on knowledge and quality of life among faculty of nursing students with vaginal infection. Obstet Gynecol Int J. 2019;10(I):I5-29. DOI: I0.15406/ogij.2019.10.00408 
Table 10 Relation between total knowledge and their total quality of life (mean and standard deviation) among students with their pre and post educational program $(n=214)$

\begin{tabular}{|c|c|c|c|c|c|c|c|c|c|c|c|}
\hline \multirow{3}{*}{ Item } & \multicolumn{9}{|c|}{ Total knowledge Scores } & \multirow{3}{*}{$\begin{array}{l}\mathbf{F} \\
\text { Test }\end{array}$} & \multirow{3}{*}{ P-Value } \\
\hline & \multicolumn{3}{|l|}{ Pretest } & \multicolumn{3}{|c|}{ Post I Month } & \multicolumn{3}{|c|}{ Post 3 Months } & & \\
\hline & Poor & Average & Good & Poor & Average & Good & Poor & Average & Good & & \\
\hline $\begin{array}{l}\text { Total quality } \\
\text { of life }\end{array}$ & $79.5 \pm \mid 4.1$ & $79.9 \pm 12.2$ & $88.1 \pm 8.8$ & $84.0 \pm 11.1$ & $84.2 \pm 13.3$ & $86.3 \pm 10.1$ & $86.4 \pm 14.2$ & $86.6 \pm 11.3$ & $87.0 \pm 11.7$ & I.545 & $0.06 \mathrm{NS}$ \\
\hline
\end{tabular}

NS, No Significant difference

Table I I Correlation between total quality of life, total knowledge and total practices scores among students pre and post educational program ( $\mathrm{n}=2 \mathrm{I} 4$ )

\begin{tabular}{|c|c|c|c|c|c|c|}
\hline \multirow{3}{*}{ Item } & \multicolumn{6}{|c|}{ Total quality of life } \\
\hline & \multicolumn{2}{|c|}{ Pretest } & \multicolumn{2}{|c|}{ Post I Month } & \multicolumn{2}{|c|}{ Post 3 Months } \\
\hline & $\mathbf{R}$ & $P$ - value & $\mathbf{R}$ & P - value & $\mathbf{R}$ & $P$ - value \\
\hline Total knowledge Scores & 0.093 & .174 NS & 0.101 & .143 NS & 0.019 & $.786 \mathrm{NS}$ \\
\hline Total Practice Scores & 0.333 & $.000 * *$ & 0.361 & $.000 * *$ & 0.31 & $.000 * *$ \\
\hline
\end{tabular}

NS, No Significant difference

** Correlation is significant at the 0.01 level.

\section{Discussion}

Vaginal infection is an important women's health problem associated with negative impacts on quality of life and has a tendency of increasing prevalence worldwide. Self-care has a key role in preventing genital infections. Early recognition of vaginal infections, initiating appropriate treatment and taking necessary precautions are essential in protecting and improving women's health. Nurses have the responsibility to educate patients related to various aspects about vaginal infection and keep themselves free from it. ${ }^{7}$

Because of the importance of this issue, female students have been selected to be studied as they are the future mothers. The current research aimed to evaluate the effect of self-care guidelines on quality of life, knowledge and practices among faculty of nursing students with vaginal infection. Research hypothesis was that "self-care guidelines will improve the quality of life, knowledge and practices of students with vaginal infection more than before implementing self-care guidelines". Quasi experimental research design was used. The results of the current study will be discussed and compared with other related studies, literature, as well as representing the researcher interpretation of the current results.

As regard socio-demographic characteristics, the present study showed that, the mean age of students was $19.9 \pm 1.1$ years and more than one third of students at $2^{\text {nd }}$ academic level. More than three quarters of students were living in rural areas and the majority of students were single. As regard mother's education, it is obvious that, more than one third was illiterate. In relation to socioeconomic status the majority of students had moderate income.

The findings of the current study agree with, Youness EM et al., ${ }^{8}$ who studied "Effectiveness of planned educational program on vaginitis and its preventive measures on adolescent female nursing student's knowledge", showed that, the mean age of studied students was $19.2 \pm 0.53$ years and the majority of them $(97.5 \%)$ were unmarried. Moreover, the findings of the current study are in agreement with Abd El-Salam AA et al., " who studied "The efficacy of learning package regarding vaginal infection and associated risk health behaviors among female university students", illustrated that, (87.9 \%) of female students were recruited at age group from (18-20) years old, with mean age of (18.69 \pm 1.20$)$. In addition, (73.5\%) were unmarried. Moreover, in agreement with Khedr NF et al., ${ }^{10}$ who studied "Vaginal secretions among students in the Egyptian universities: prevalence, knowledge and practices" more than two thirds of students' age was ranged from $21-23$ years old $(71.9 \%)$ and $(68.2 \%)$ were from rural areas.

Regarding the prevalence of vaginal infection among nursing students, the findings of the present study showed that, about one quarter $(25 \%)$ suffer from vaginal infection from the total number of female students in the faculty. The findings of the current study are in contrast with the study conducted by Youness EM et al., ${ }^{8}$ in Al-Fayoum University which revealed that the prevalence of vaginal infection among faculty of nursing students was $(76.0 \%)$. Moreover, the finding of the current study in contrast with the study conducted by Khedr NF et al., ${ }^{10}$ in Mansoura University which revealed that the prevalence of vaginal infection among girls at Egyptian universities in the camp was $(53.4 \%)$.

Also, the findings of the current study are in contrast with the study conducted by Emam WM et al., ${ }^{11}$ in Ain Shams University revealed that, the prevalence of abnormal vaginal discharge among faculty of nursing students was $(60.6 \%)$. The findings of the current study are in contrast with the study conducted by Mohamed HA et al., ${ }^{12}$ in Benha University revealed that, the prevalence of vaginal infection among faculty of nursing students was $(65.7 \%)$. The findings of the current study may be interpreted due to embarrassment of female students to participate in the present study due to culture difference in Upper Egypt.

Regarding distribution of the studied students according to their symptoms of vaginal infection, the present study observed that more than one third of students suffer from candidiasis signs and only $(3.7 \%)$ suffer from bacterial vaginosis. The findings of the present study are in the line with the study conducted by Hamed $A G,{ }^{13}$ who studied "The impact of genital hygiene practices on the occurrence of vaginal infection and the development of a nursing fact sheet as 
prevention massage for vulnerable women" revealed that the white cheese like discharge was the most common (candida signs) $(45 \%)$, and $(4.8 \%)$ from bacterial vaginosis.

Moreover, the findings of the current study are in agreement with Khedr NF et al. ${ }^{10}$ who revealed that, $(32.7 \%)$ suffer from candidiasis signs and (5.8\%) suffer from bacterial vaginosis. Also, the findings of the current study in agreement with Emam WM et al., ${ }^{11}$ who studied "Effect of vaginal discharge on nurses student's quality of life" showed that, about (46.4\%) thick white cheese like discharge (candida signs) and (9.2\%) bacterial vaginosis.

Moreover, the findings of the current study are in agreement with the study conducted by JA Lennox et al., ${ }^{14}$ who studied "The prevalence of vaginitis and vaginosis among female students in University of Calabar" revealed that, about (64.71\%) had candidiasis vaginitis while only $(35.29 \%)$ had bacterial vaginosis. The findings of the current study may be due to exposure of young female to risk factors of candidiasis such as wearing tight non-cotton underwear for long time, using antibiotic continuously, frequent using of vaginal deodorants or perfumed soaps (alkaline medium), stress and lack of sleep.

In addition, the findings of the present study are in contrast with Juan F et al., ${ }^{15}$ who studied "Risk factors associated to vaginal infections and squamous intraepithelial lesions in university students in Medellín, Colombia”, illustrated that $(29.5 \% \%)$ had bacterial vaginosis and $(11.4 \% \%)$ were positive by Candida. That finding may be due to increase bacterial vaginosis with multiple sex.

Additionally the period of vaginal infection symptoms from the beginning at this episode around two thirds of students the symptoms last less than one week. On the other hand, around two thirds of students the symptoms occur about three times or more per year, and more than half of students $(57.0 \%)$ not treated and can't tell any person. The finding of the current study in partial agreement with study conducted by Masoumeh E et al., ${ }^{2}$ who studied "Assessment of educational needs among women of reproductive age with common genital tract infections (vaginitis): the first step for developing a selfcare educational package", revealed that, duration of symptoms were with an average of $22.7 \pm 24.5$ day, and symptoms occurs with an average of $2.8 \pm 2.6$ times per year, and with an average of $1.7 \pm 1.4$ time treatment visits during the preceding year.

Moreover, the findings of the current study are in line with findings of the study conducted by Sabarwal et al., ${ }^{16}$ who studied "Treatment seeking for symptoms of RTIs among young women" found that, the treatment seeking for any RTI infection was poor and $66 \%$ of unmarried women had not sought any treatment for their symptoms. Correspondingly, the finding by Prusty \& Unisa ${ }^{17}$ who studied "RTIs and treatment-seeking behavior among married female adolescents in India", found that, treatment-seeking behavior among female adolescents is poor. The findings of current study interpreted that in spite the recurrence of infection per year many times for female students they didn't seek treatment due to many barriers.

Meanwhile, there are barriers that prevent female students from seeking gynecologist when exposed to vaginal infection symptoms. It was observed that the shyness was the most pronounced barrier $(58.0 \%)$. The belief that this is a natural thing comes the second barrier (28.5\%), This result is in agreement with Khedr NF et al., ${ }^{10}$ who revealed that, shamed from exposure of genital area and thought that vaginal discharge was simple thing and didn't need going to physician were the most prominent reasons for didn't consult doctor which constituted $(57.8 \%$ and $48.9 \%)$ respectively. Moreover, in partial agreement with Abdelnaem S et al., ${ }^{18}$ who studied "Perception of late adolescent girls about sexually transmitted diseases and infections in El-Minia University", observed that, the shyness was the most pronounced barrier (38.2\%). Tradition come the second barrier $(21.1 \%)$, followed by having no special girls' center $(10.3 \%)$, and $(4.9 \%)$ not need to seek health care.

As regards to the sources of knowledge about vaginal infection, the finding of the present study notices that more than half $(59.8$ $\%$ ) selected the mother as a source of information about infection, followed by friends \& relatives $49.1 \%$, mass media such as T.V and internet $39.3 \%$, Physician $27.1 \%$, Nurse $25.7 \%$, school, university, books $23.8 \%$, and from others sources as (pharmacist) $9.8 \%$. This interpreted the importance of increasing mother's level of knowledge regarding all issues related to sexual health because they play a crucial role in delivering any message related to RTI.

The findings of the current study are supported by Mohamed HA et al., ${ }^{12}$ who showed that, $(43 \%)$ of the studied students seek advice from their mothers, while $(29.0 \%)$ of them mentioned physician as a source of their health advice, $(26.0 \%)$ of them mentioned friend or sister and only $(2 \%)$ of students mentioned nurse as a source of their health advice. Moreover, the finding of the current study is supported by Youness EM et al., ${ }^{8}$ who showed that the most common source of knowledge was their friends and their family members in $80.0 \%$ and $66.6 \%$, respectively.

However the finding of the current study is in contrast with the study conducted by Sevil S et al., ${ }^{19}$ who studied "An evaluation of the relationship between genital hygiene practices, genital infection", revealed that, nearly half of the students described that healthcare professionals were their preferred source of information. This may be interpreted due to cultural difference. Additionally, in contrast with Sarah R et al., ${ }^{20}$ who studied "Attitudes and experience of women to common vaginal infections", showed that, women prefer to seek advice from physician/nurse to correct diagnosis and trust in their expert opinion. Also, in contrast with Renju, ${ }^{21}$ revealed that the most common source mass media, health personnel and family member $11.3 \%, 7.5 \%$ and $6.3 \%$ respectively. These findings may be interpreted due to culture difference.

Regarding distribution of the students according to their total knowledge score about vaginal infection. The finding of the present study showed that the mean of knowledge score in post-test was higher than the mean of pre-test knowledge score. There was a significant improvement in total knowledge about vaginal infection among students in posttest as compared to pretest assessment $(p<0.001)$. Hence, the finding of present study interpreted that implementing selfcare guidelines regarding vaginitis and its preventive strategies was effective in increasing the level of students' knowledge.

Regarding distribution of the students related to their total knowledge score about vaginal infection, the finding of the current study showed that there was a significant improvement in total knowledge about vaginal infection among students in posttest as compared to pretest assessment $(p<0.001)$. Hence, the finding of the current study interpreted that implementing self-care guidlines regards vaginitis and its preventive strategies was effective in increasing the level of students' knowledge. The finding of the current study is in agreement with Youness EM et al., ${ }^{8}$ who revealed that as regards the total knowledge score level, the mean of post-test knowledge score $(8.3 \pm 0.8)$ of the studied students was higher than that of the mean pretest knowledge score $(3.5 \pm 2.6)$. There was a statistically 
significant difference $(\mathrm{P}<0.001)$ as regards the pretest and post-test total knowledge score level among the studied students.

Moreover, the finding of the current study is supported by Alka et al., ${ }^{22}$ who studied "Effectiveness of a "planned teaching programme" (ptp) on knowledge related to reproductive tract infections among rural women", They found that, the mean of post-test knowledge score $(16.93 \pm 3.70)$ was higher compared with the mean of pretest knowledge score (13.25 \pm 3.71$)$. There was a significant difference between the mean pretest and post-test scores at $\mathrm{P}<0.05$ levels.

Likewise, the findings reported by Yarmohammadi et al., ${ }^{23}$ who studied "The effect of education on knowledge, attitude, and practices of patients with vaginitis", revealed that, the mean of post-test knowledge score $(1.8 \pm 0.35)$ was higher compared with the mean of pretest knowledge score $(1.1 \pm 0.23)$ and indicated a significant increase in the mean score of knowledge, attitude, and practice of patients in the intervention group. Moreover, the findings of the current study agree with the study conducted by Pratibha $\mathrm{K},{ }^{24}$ "An awareness program on prevention of vaginal candidiasis among pregnant women" who indicated that, mean percentage of post-test knowledge score $(10.97 \pm 1.77)$ was higher than the mean percentage of pretest $(5.74 \pm 2.25)$. There was significant difference between the mean pretest and post-test knowledge scores of the samples $(\mathrm{p}=<0.001)$.

Regarding distribution of students related to their total practice regarding vaginal infection, the finding of the current study noticed that there was a significant improvement in total practice regarding vaginal infection among students in post-test as compared to pretest assessment $(\mathrm{p}<0.001)$. The finding of the current study interpreted that after the implementation of educational program, there was a positive effect on their practices. Most of the students with vaginal infection symptoms have been significantly relieved or somewhat improved after healthy self-care measures have been instructed, followed and used $(p<0.001)$. The finding of the current study is in agreement with Youness EM et al., ${ }^{8}$ who illustrated that, the mean of post-test score $(8.3 \pm 0.8)$ of the studied students was higher than that of the mean pretest score $(3.5 \pm 2.6)$ in relation to general hygienic measures that prevent vaginitis. The scores predicted that there was a significant difference between the mean of pretest and post-test at $\mathrm{P}$ less than 0.001 levels.

Moreover, the finding of the current study agree with Abd El-Salam AA et al., ${ }^{9}$ revealed that, there were statistical significant deference among the female students regarding the preventive measures of genital infection in pre and post intervention ( $p$ value $<0.001$ ). In addition, the findings of the current study agree with Soudabeh $Y,^{25}$ who studied "The effect of education on knowledge, attitude and practice of patients with vaginitis" showed a significant difference in the mean of performance score before $(2.61 \pm 0.22)$ and after $(2.81 \pm 0.35)$ intervention ( $\mathrm{p}$ value $=0.002)$.

Regarding distribution of the students according to their total quality of life, the current study revealed that the mean score of total quality of life at post-test was higher than the mean score at pretest. There was a significant improvement in total QOL among students in post-test as compared to pretest assessment $(\mathrm{p}<0.001)$. Hence, the finding of the current study is interpreted that self-care guideline regarding vaginitis was effective in improving the QOL of students.

The finding of the current study is supported by Sameer V et al., ${ }^{26}$ who studied "Impairment of quality of life in symptomatic reproductive tract infection and sexually transmitted infection" showed that, there was a significant differences between the domains as well as the total QOL score before (33.4 \pm 3.45$)$ and after $(56.07 \pm 3.31)$ intervention. So, QOL measures give more direct measure of the impact of the disease on daily life and this is more relevant in RTI/STIs where the condition is distressing physically, psychologically and treatment seeking is hindered by numerous factors which are predominantly social.

Moreover, the present study is supported with Roxana $\mathrm{P}$ et al., ${ }^{27}$ who studied "Impact of health-promoting educational intervention on lifestyle (nutrition behaviors, physical activity and mental health) related to vaginal health among reproductive-aged women with vaginitis", showed that, the mean of total health-promoting lifestyle scores at pre-intervention and post-intervention were statistically significant difference $(\mathrm{P}<0.001)$. Therefore, the educational intervention focused on health promoting lifestyle behaviors are a valuable way to reduce the incidence and impact of health problems, reduce health care costs, and improve quality of life.

Also, the finding of the current study is in agreement with a study conducted by Mirghafour V et al., ${ }^{28}$ who studied women of reproductive age in Tehran. Their studies have reported a relationship between self-efficacy and the sub-domains of health related lifestyles such as nutrition, physical activity, health responsibility, and other sub-domains of QOL. Also, the current study is in the same line with Samuel A et al.," ${ }^{29}$ who studied "Subjective health status and healthrelated quality of life among women with Recurrent Vulvovaginal Candidosis (RVVC) in Europe and the USA" mentioned that, SF36 QOL dimensions were all affected among women with RVVC in general. The greatest impact of the disease was found on the emotional domains, but significant differences were found for other domains as well. The summary scores of the SF-36 also suggested that mental health was strongly affected by RVVC.

In addition, the finding of the current study is consistent with the results from EQ-5D findings by Mendling et al., ${ }^{30}$ who also reported a stronger impact on mental health than on physical health using SF36, albeit to a somewhat lesser extent. Moreover, the finding of the current study is supported by Jade E et al.," "The burden of bacterial vaginosis: women's experience of the physical, emotional, sexual and social impact of living with recurrent bacterial vaginosis" mentioned that, recurrent bacterial vaginosis had impact on physical, emotional, sexual, social life and total QOL.

Regarding the relationship between socio-demographic data of the students with their total knowledge, the finding of the current study noticed that there was a highly statistically significant relation between the student's total score of pretest knowledge as regards vaginitis with their age, academic level, residence and mother's education ( $\mathrm{P}$ value $<0.001,0.001,0.02$, and, 0.04 , respectively). While in post-test after 1 month there was no statistically significant $(p>0.05)$ relation between the studied students' total knowledge score with students socio-demographic. After 3 months it was noticed that there was a highly statistically significant relation between the students' total knowledge score with their age and academic level only $(\mathrm{P}<0.005$, 0.01 , respectively).

The finding of the current study supported by Emam WM et al., ${ }^{11}$ revealed that there is a highly significant relation between student's knowledge means score about VD and their age, place of residence, and academic year. Also, this finding is supported by Youness EM et al., ${ }^{8}$ showed that, there was a highly statistically significant relation between students' total score of pretest knowledge as regards vaginitis with their residence, and mother's education $(\mathrm{P}<0.002$, and 0.001 , respectively). Furthermore, the current study is in agreement with 
the findings of Mohamed HA, ${ }^{12}$ who revealed that there was a highly statistically significant relation between students' total knowledge score level and their residence and marital status. This can be attributed to lack of awareness in rural areas as compared with the urban areas. These findings are in agreement with the findings of Chauhan et al., ${ }^{32}$ who concluded that there was a highly statistically significant relation between students' total knowledge score level and their age, education and marital status. Hence, it is interpreted that there is an association between demographic variables with pretest knowledge score and the impact of program on the level of knowledge of students.

However, the current study were incongruent with the findings of Renju, ${ }^{21}$ who found that there was no association between pretest knowledge score and selected demographic variables. Moreover, the finding of the current study in contrast with the study conducted by Pratibha $\mathrm{K},{ }^{24}$ as Chi-square was computed between pre-test knowledge scores and selected demographic variables showed that there was no significant association between pre-test knowledge scores and selected demographic variables.

Regarding the relationship between socio-demographic data of the students with their total practices, it was noticed that there was a highly statistically significant relation between the students' total practices score of pretest with their age and academic level ( $\mathrm{P}$ value $<0.03$ and 0.002 , respectively). This might be explained that, increasing students' age may have higher level of awareness about proper hygienic practices in order to maintain a healthy reproductive tract that is needed in the childbearing period. While in post-test after 1 month and 3 months, it was noticed that, there was no statistically significant $(p>0.05)$ relation between the studied student's total practices score with student's socio-demographic data.

The finding of the current study is in contrast with Mohamed HA et al., ${ }^{12}$ who showed that, there was a statistical significant relation between students' total practice score level with their residence, their marital status and their mother education. However, in contrast with the finding by Slave et al. ${ }^{33}$ who found that, there was statistical significant relation between hygienic practices among adolescent girls with their residence. Also, against Busari, ${ }^{34}$ who found that, there was statistically significant relation between students' hygienic practices with the level of their mother education.

As regarding the relation between socio-demographic data of the students with their quality of life (QOL), the finding of the current study showed that there was a highly statistically significant relation between the student's total quality of life score of pretest with their socioeconomic status $(\mathrm{P}<0.01)$. In post-test after 1 month it was noticed that there was statistically significant relation between the students' total QOL score with their academic level and socioeconomic status ( $\mathrm{P}$ value $<0.001$ and 0.001 , respectively). In post-test after 3 months, it was noticed that, there was statistically significant relation between the students' total QOL score with their socioeconomic status $(\mathrm{P}<0.01)$. The finding of the current study might be explained that, the low socio- economic standards play a major role on the ability of buying medications that relieving symptoms of infection and improving QOL.

The finding of the current study disagree with Emam WM et al.," who studied "Effect of vaginal discharge on nurses student's quality of life" showed that, there was a highly significant relation between students' score about QOL with their age and place of residence.

Regarding the relation between total knowledge of the students with their total practices, the finding of the current study showed that there was highly statistical significant relation between total knowledge of the students with their practices ( $\mathrm{P}$ value $<0.01$ ). So, implementation of self-care guideline regarding vaginal infection was effective as a method to improve the knowledge, practices of adolescent nursing students as regards vaginitis. The finding of the present study is in agreement with Abd El-Salam AA et al., ${ }^{9}$ who revealed that, there were highly statistical significant deference among the female students total post-practice and total post-knowledge score level regarding post intervention ( $\mathrm{p}$ value $<0.001$ ).

Furthermore, the finding of the present study is in agreement with Mohamed HA et al., ${ }^{12}$ who studied "The health practices among female students at Benha University as regards prevention of RTIs" showed that, there was positively correlation between having satisfactory/unsatisfactory knowledge and healthy/unhealthy practices, Thus, when knowledge improves, practice tend to be more healthy (hygienic).

In addition, the finding of the present study is in agreement with Bobhate \& Shrivastava, ${ }^{35}$ who studied "Across sectional study of knowledge and practices about reproductive health among female adolescents in urban mumbai" mentioned that, there was significant association between having good knowledge and good practice regarding reproductive tract infection. Moreover, the finding of the current study in the same line with the finding by Soudabeh Y., ${ }^{25}$ who studied "The effect of education on knowledge, attitude and practice of patients with vaginitis", showed that, the mean scores of knowledge and performance of the study group after the program was increased, indicating a significant effect on the level of awareness in women's behavior and practice. So, teaching programs about health can increase the level of knowledge and attitude changing into better practice.

Regarding the relation between total quality of life; total knowledge total QOL of the students. The finding of the present study observed that there was no statistical significant relation between total knowledge of the students with their total quality of life. However, there was positive correlation between their quality of life and their practices. The finding of the present study is in partial agreement with Emam WM et al., ${ }^{11}$ who studied "Effect of vaginal discharge on nurses student's quality of life" There was a statistical significant difference between nursing students' knowledge regarding vaginal discharge and their quality of life. There was highly statistical significant difference between nursing students' quality of life and self-care practices regarding vaginal discharge.

\section{Conclusion}

The current study concluded that there were highly statistical significant correlation between total knowledge of the students and their practices $(\mathrm{P}<0.001)$. Moreover, there was statistical significant correlation between practices of the students and their quality of life $(\mathrm{P}<0.001)$. So, implementation of self-care guideline about vaginal infection was effective in improving the knowledge, practices and the quality of life for nursing students with vaginal infection.

\section{Recommendations}

a) Applying educational programs for adolescent females and their mothers to increase awareness about vaginal infection.

b) Develop a special health center for adolescent females that motivate them to seek prevention, early diagnosis and treatment of vaginal infection that may have positive impact on their future health and their QOL. 
c) Evaluate and develop strategies to improve factors facing adolescent students to use reproductive health services.

d) More studies and researches needed to investigate other risk factors for acquiring vaginal infection and the relationships between genital hygiene and genital infections.

\section{Acknowledgments}

The researchers would like to acknowledge the contribution of all participants who kindly agreed to be a part of this work.

\section{Conflicts of interest}

The authors declare no conflicts of interest.

\section{References}

1. Ram R, Bhattacharya SK, Bhattacharya K, et al. Reproductive tract infection among female adolescents. Indian $J$ Comm Med. 2006;31(1):32-33.

2. Ebrahimi M, Ghofranipour F, Hajizadeh E, et al. Assessment of educational needs among women of reproductive age with common genital tract infections (vaginitis): the first step for developing a self-care educational package. International J Women's Health and Reproduction Sciences. 2015;3(4):201-207.

3. Parsapure R, Rahimiforushani A, Majlessi F, et al. Impact of healthpromoting educational intervention on lifestyle (nutrition behaviors, physical activity and mental health) related to vaginal health among reproductive-aged women with vaginitis. Iran Red Crescent Med J. 2016;18(10):e37698

4. Dehdari T, Ramezankhani A, Zarghi A. Principles of Health Promotion. Tehran: Nazari Publication; 2011.

5. Johnson SR, Griffiths H, Humberstone FJ. Attitudes and experience of women to common vaginal infections. J Low Genit Tract Dis. 2010;14(4):287-294.

6. AlexandraR,ElWerdanyM,HadouraE, etal.VaginalDischarge.Obstetrics, Gynaecology \& Reproductive Medicine. 2016;26(11):317-323.

7. Workowski K, Berman S. Sexually transmitted diseases treatment guidelines. Centers for Disease Control and Prevention. 2010;59(RR12):1-110.

8. Youness EM, Ayat Masoud Omar. Effectiveness of planned educational program on vaginitis and its preventive measures on adolescent female nursing student's knowledge. Egyptian Nursing J. 2017;14(1):1-8.

9. Abd El-Salam AA, Abeer Mohamed Emaghwery Eldeeb, Fatma zaki Frahat. The efficacy of learning package regarding vaginal infection and associated risk health behaviors among female university students. The Malaysian J Nursing. 2018;9(4):84-94.

10. Khedr NF, Hanan Awad Mohamed, Elmashad and Adel Al Wehedy. Vaginal secretions among students in the Egyptian Universities: prevalence, knowledge and practices. World $J$ Nursing Sciences 2015;1(3):68-75.

11. Emam WM, Shadia Hamedo Mohaseb, Randa Mohamed Ibrahim. Effect of vaginal discharge on nurses student's quality of life. Ain Shams University; 2015.

12. Mohamed HA, Amira A El-Beih, Ebtisam M. Abd-ElAal. Health practices among female university students regarding prevention of reproductive tract infections. Benha University; 2013.

13. Hamed AG. The Impact of Genital Hygiene Practices on the Occurrence of Vaginal Infection and the Development of a Nursing Fact Sheet as Prevention Massage for Vulnrable Women. OSR J Nursing and Health Science. 2015;4(6):55-64.
14. Lennox LA, Abbey SD, Udiba D, et al. Prevalence of vaginitis and vaginosis among University of Calabar female students. J Public Health and Epidemiology. 2013;5(4):167-172.

15. Zapata Martínez JF, Pérez Muñoz A, Tirado Otálvaro AF, et al. Risk factors associated to vaginal infections and squamous intraepithelial lesions in university students in Medellín, Colombia. Enfermería Global. 2017:97-107.

16. Sabarwal S, Santhya KG. Treatment-seeking for symptoms of reproductive tract infections among young women in India. Int Perspect Sex Reprod Health. 2012;38:90-98.

17. Prusty RK, Unisa S. Reproductive tract infection and treatment seeking behavior and married Adolescent women in India". International Institute for Population Science, Deonar, Mumbai, 400088, India. Int $J \mathrm{MCH}$ AIDS. 2013;2(1):103-110.

18. Abd Elnaem Safaa A, Manal F Moustafa, Ekbal A Emam. Perception of Late Adolescent Girls about Sexually Transmitted Diseases and Infections in El-Minia University. Assiut Scientific Nursing J. 2010;1(2).

19. Sevil S, Kevser O, Aleattin U, et al. An Evaluation of the Relationship between Genital Hygiene Practices, Genital Infection. Gynecol Obstet. 2013;3:187.

20. Sarah R Johnson, Heather Griffiths, Fiona J Humberstone. Attitudes and Experience of Women to Common Vaginal Infections. J Low Genit Tract Dis. 2010;14(4):287-294.

21. Renju S. Effectiveness of planned teaching program (PTP) on vaginitis and its prevention among adolescents of selected pupil colleges in Mangalore [dissertation], Bangalore, Karnataka, India. Rajiv Gandhi University of Health Sciences; 2010.

22. Alka Chauhan, Divya Chawla, Garima Saini, et al. Effectiveness of a Planned Teaching Programme (PTP) on Knowledge Related to Reproductive Tract Infections Among Rural Women. J Nursing and Health Science. 2014;3(1):17-21.

23. Yarmohammadi S, Taheri G, Mousavi SS, et al. The effect of education on knowledge, attitude and practice of patients with vaginitis. Adv Biol Res. 2015;9:196-200.

24. Pratibha K, Maria P, Malathi $G$, et al. An awareness program on prevention of vaginal candidiasis among pregnant women. NUJHS. $014 ; 4(2)$.

25. Soudabeh Y, Taheri G, Mousavi SS, et al. The effect of education on knowledge, attitude and practice of patients with vaginitis. Advances in Biological Research. 2015;9(3):196-200.

26. Valsangkar S, Selvaraju D, Rameswarapu R, et al. Impairment of quality of life in symptomatic reproductive tract infection and sexually transmitted infection. J Reprod Infertil. 2014;15(2):87-93.

27. Parsapure R, Rahimiforushani A, Majlessi F, et al. Impact of HealthPromoting Educational Intervention on Lifestyle (Nutrition Behaviors, Physical Activity and Mental Health) Related to Vaginal Health Among Reproductive-Aged Women With Vaginitis. Iran Red Crescent Med J. 2016;18(10):e37698.

28. Mirghafourvand M, Baheiraei A, Nedjat S, et al. A population-based study of health-promoting behaviors and their predictors in Iranian women of reproductive age. Health Promot Int. 2015;30(3):586-594.

29. Aballéa S, Guelfucci F, Wagner J, et al. Subjective health status and health-related quality of life among women with Recurrent Vulvovaginal Candidosis (RVVC) in Europe and the USA. Health Qual Life Outcomes. 2013;11:169.

30. Mendling W, Birkner V. Vaccination with inactivated Lactobacilli or heliotherapy can improve the qualityof-life of women with chronic recurrent vulvovaginal candidosis - a prospective randomized study. Geburtsh Frauenheilk. 2011;71(9):767-772. 
31. Bilardi JE, Walker S, Temple-Smith M, et al. The Burden of Bacterial Vaginosis: Women's Experience of the Physical, Emotional, Sexual and Social Impact of Living with Recurrent Bacterial Vaginosis. PLoS One. 2013;8(9):e74378.

32. Chauhan A, Chawla D, Saini G, et al. Effectiveness of a planned teaching program (PTP) on knowledge related to reproductive tract infections among rural women. IOSR J Nurs Health Sci. 2014;3(1):17-21.

33. Slave S, Dase R, Mahjan S, et al. Assessment of Knowledge \& Practices about Menstrual Hygiene amongst Rural \& Urban Adolescent Girls: A comparative Study. International J Recent Trends in Science \& Technology. 2012;3(3):65-70.
34. Busari A. Menstrual Knowledge \& Health Care Behavior among Adolescent Girls in Rural Nigeria. International J Applied Science and Technology. 2012;2(4):150-153.

35. Bobhate P, Shrivastava S. Across Sectional Study of Knowledge \& Practices about Reproductive Health among Female Adolescents In urban Mumbai. J Family \& Reproductive Health. 2011;5(4):117-124. 